\title{
Peripartum cardiomyopathy: An uncommon cause of ischemic stroke
}

\author{
Miocardiopatía periparto: una causa infrecuente de enfermedad vascular cerebral \\ isquémica
}

\begin{abstract}
Amado Jiménez-Ruiz¹, Miguel García-Grimshaw ${ }^{1 *}$, Jorge Ortega-Márquez², Alejandro Moreno-Guillen³, Fernando D. Flores-Silva ${ }^{1}$, and Carlos Cantú-Brito ${ }^{1}$

${ }^{1}$ Department of Neurology and Psychiatry, Instituto Nacional de Ciencias Médicas y Nutrición Salvador Zubirán; ${ }^{2}$ Facultad de Medicina, Universidad Nacional Autónoma de México; ${ }^{3}$ Department of Cardiology, Centro Médico ABC. Mexico City, Mexico
\end{abstract}

A previously healthy 22-year-old woman with a history with a healthy pregnancy presented 12 weeks after an uneventful delivery with progressive dyspnea and edema. Initial blood workup was relevant for an elevated B-type natriuretic peptide in $1641 \mathrm{pg} / \mathrm{mL}$ (reference: $0-100)$. Her electrocardiogram was unremarkable, and a chest $\mathrm{X}$-ray revealed cardiomegaly with bilateral pleural effusion (Fig. 1A). A transthoracic echocardiogram (TTE) and a cardiac magnetic resonance imaging (MRI) showed generalized hypokinesia, a severely dilated left ventricle (LV) with reduced ejection fraction ( $\mathrm{rEF}$ ) of $28 \%$ and multiple mobile intracavitary thrombi (Fig. 1B and C), confirming a diagnosis of peripartum cardiomyopathy (PPCM). We started treatment with furosemide, dobutamine, and anticoagulation with nadroparin and warfarin. Three days after admission, we added treatment with metoprolol, spironolactone, sacubitril/valsartan, and gradually weaned dobutamine off.

On day 5 of in-hospital stay, she awoke with right-sided hemiparesis, and a brain MRI showed multiple supra and infratentorial ischemic lesions (Fig. 2), with no diffusion-weighted imaging/fluid-attenuated inversion recovery mismatch (DWI/FLAIR), consistent with acute cardioembolic strokes. We discharged her 12 days after

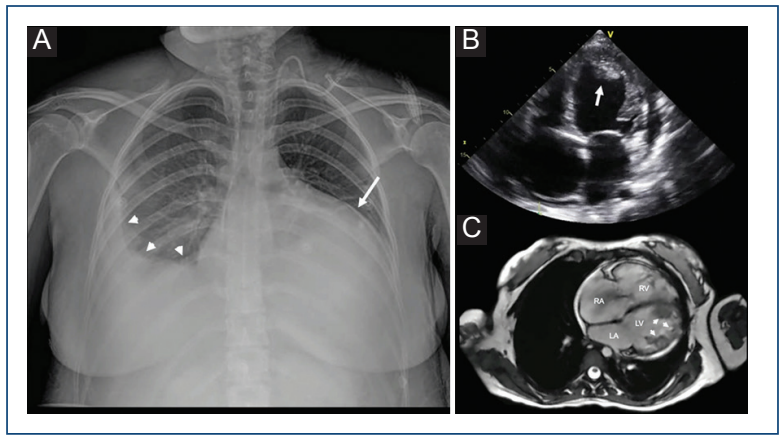

Figure 1. A: chest X-ray shows cardiomegaly (arrow) and bilateral pleural effusion (arrowheads). B: transthoracic echocardiogram shows a left ventricle (LV) mobile intracavitary thrombus (arrow). C: cardiovascular magnetic resonance imaging shows a severely dilated heart with multiple intracavitary thrombi in the LV (arrows). LA: left atrium; LV: left ventricle; RA: right atrium, RV: right ventricle.

admission on warfarin, spironolactone, furosemide, metoprolol, and sacubitril/valsartan. Six months after the event, she remained with right-sided hemiparesis, and a follow-up TTE showed resolution of the intracavitary thrombi without improvement of the LV dysfunction.

\section{Correspondence:}

*Miguel García-Grimshaw

E-mail: miguelgrimshaw@gmail.com
Date of reception: 06-06-2020

Date of acceptance: 01-07-2020

DOI: $10.24875 /$ ACM.20000263
Available online: 12-11-2020

Arch Cardiol Mex (Eng). 2021;91(2):249-251

www.archivoscardiologia.com

1405-9940 / @ 2020 Instituto Nacional de Cardiología Ignacio Chávez. Published by Permanyer. This is an open access article under the CC BY-NC-ND license (http://creativecommons.org/licenses/by-nc-nd/4.0/). 


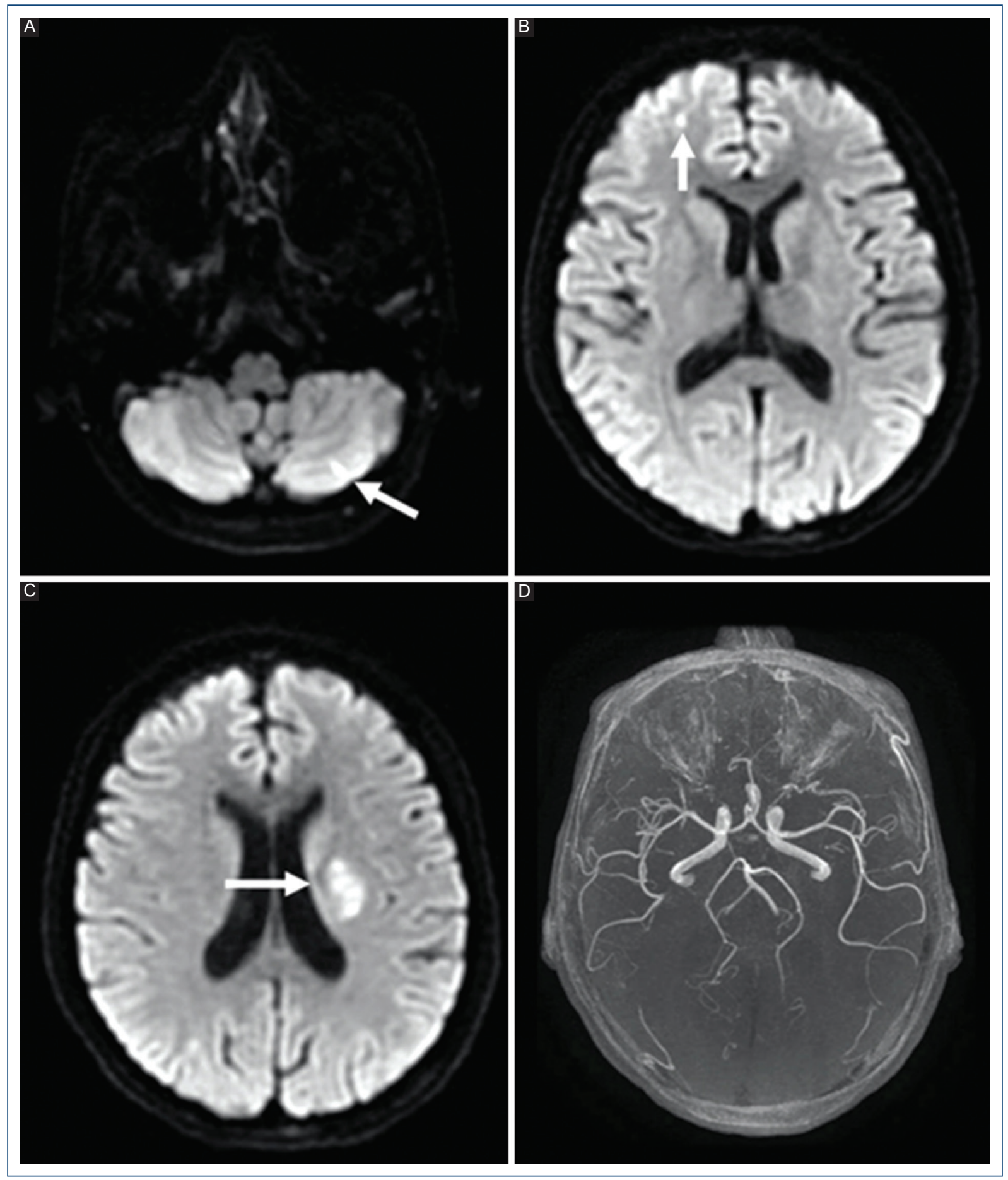

Figure 2. Axial diffusion-weighted imaging magnetic resonance imaging (MRI) of the brain shows multiple acute ischemic strokes (arrows) located in the A: left cerebellum, B: right frontal lobe, and C: left internal capsule. D: 3D time-of-flight MRI angiography shows normal intracranial vessels.

PPCM is characterized by LV dysfunction with rEF and symptoms of congestive heart failure during the end of pregnancy and early postpartum period, without an identifiable cause. It has a variable incidence between 1:100 and 1:20,000 deliveries worldwide ${ }^{1,2}$. PPCM is associated with a recovery rate of $50-80 \%$ to a normal LV systolic function (ejection fraction $\geq 50 \%$ ), usually within the first $3-6$ months, but $13 \%$ of women 
can develop persistent cardiomyopathy with rEF. Furthermore, up to $30 \%$ of patients may develop LV throm$\mathrm{bi}^{3}$. During the puerperium, selection of anticoagulation with either low-molecular-weight heparin or Vitamin $\mathrm{K}$ antagonists is recommended mainly on clinical experience gathered from other causes of heart failure ${ }^{2,3}$.

Worldwide, ischemic stroke during pregnancy and postpartum occurs in 12.2 per 100,000 pregnancies ${ }^{4}$, and in a Mexican series of 240 women, it occurred in $26.7 \%$ of patients ${ }^{5}$. The prevalence of PPCM-related stroke remains unknown ${ }^{4}$. PPCM complicated with intracavitary thrombi is an uncommon cause of stroke to acknowledge in a woman presenting with acute focal neurological signs during the last trimester of pregnancy or the early postpartum period.

\section{Conflicts of interest}

None.

\section{Funding}

The authors declare that they did not receive any sponsoring to carry out this article.

\section{Ethical disclosures}

Protection of human and animal subjects. The authors declare that no experiments were performed on humans or animals for this study.

Confidentiality of data. The authors declare that they have followed the protocols of their work center on the publication of patient data.

Right to privacy and informed consent. The authors have obtained the written informed consent of the patients or subjects mentioned in the article. The corresponding author is in possession of this document.

\section{References}

1. Schaufelberger M. Cardiomyopathy and pregnancy. Heart. 2019;105:1543-51.

2. Davis MB, Arany Z, McNamara DM, Goland S, Elkayam U. Peripartum cardiomyopathy: JACC state-of-the-art review. J Am Coll Cardiol. 2020;75:207-21

3. Arany Z, Elkayam U. Peripartum cardiomyopathy. Circulation 2016;133:1397-409.

4. Swartz RH, Cayley ML, Foley N, Ladhani NN, Leffert L, Bushnell J, et al. The incidence of pregnancy-related stroke: a systematic review and meta-analysis. Int J Stroke. 2017;12:687-97.

5. Cantu-Brito C, Arauz A, Aburto Y, Barinagarrementeria F, Ruiz-Sandoval JL, Baizabal-Carvallo JF. Cerebrovascular complications during pregnancy and postpartum: clinical and prognosis observations in 240 Hispanic women. Eur J Neurol. 2011;18:819-25. 